\title{
Immunotherapy-related skeletal muscle weakness in cancer patients: a case series
}

\author{
Amy H. Ng", Diana M. Molinares"*, An T. Ngo-Huang, Eduardo Bruera \\ Department of Palliative, Rehabilitation and Integrative Medicine, The University of Texas MD Anderson Cancer Center, Houston, TX, USA \\ \#These authors contributed equally to this work. \\ Correspondence to: Dr. Amy H. Ng. The University of Texas MD Anderson Cancer Center, 1515 Holcombe Blvd., Unit 1414, Houston, Texas 77030, \\ USA. Email: ANg@mdanderson.org.
}

\begin{abstract}
Immunotherapy has become a lifeline in cancer treatment. However, increasingly reports of programmed death-1 (PD-1) inhibitors have been linked to autoimmune disorders. We highlight two unique cases that presented with skeletal weakness and resulted in fatalities after developing de novo myasthenia gravis and myositis associated to immunotherapy treatment. Additionally, we describe the importance of early symptoms recognition and prompt treatment in preventing prolonged functional impairments and decreasing associated mortality. Patient 1: a 65-year-old male with a history of metastatic clear cell renal cell cancer with metastasis to the lungs presented with unusual neck weakness, double vision, trouble breathing, and symptoms of urinary incontinence after his $4^{\text {th }}$ cycle of nivolumab (480 mg every 4 weeks). He had a positive fatigability test, diplopia, and proximal weakness in bilateral upper extremities with an abnormal negative inspiratory force (NIF). Patient 2: a 83 -year-old male with history of metastatic urothelial carcinoma presented with generalized weakness, difficulty holding his head up and swallowing three days after receiving the second cycle of immunotherapy treatment with nivolumab (480 mg every 4 weeks). He had proximal muscle weakness but normal sensation, fatigability tests and reflexes, very abnormally high creatine kinase (CK) greater than 4,000 ng/L, EMG results of myopathy and muscle biopsy showing focal perimysial chronic inflammatory cell infiltrates. Patient 1 ultimately died due to acute hemorrhage while patient 2 had a prolonged hospitalization and rehabilitation and ultimately discharged home with hospice. Prompt symptom recognition and treatment can potentially prevent prolonged impairment and mortality associated with cancer immunotherapy.
\end{abstract}

Keywords: Cancer; case report; immunotherapy; myasthenia gravis; myositis

Submitted Feb 19, 2020. Accepted for publication May 15, 2020.

doi: 10.21037/apm-20-454

View this article at: http://dx.doi.org/10.21037/apm-20-454

\section{Introduction}

Over the past couple of decades, anti-cancer drug research has focused on the development of therapies that use a patient's immune system to treat cancer, and immunologists have been working on identifying new ways to trigger an anti-tumor immune response. The 2018 Nobel Prize in Physiology or Medicine recognized the importance of tumor immunology by recognizing Drs. Allison and Honjo for their research on the T-lymphocyte-associated protein 4 (CTLA-4) and programmed cell death protein 1 (PD-1) pathways, respectively (1). Targeted therapies are now offered in many types of cancers with hope they will work equally well if not better than chemotherapy, as immunotherapy acts on specific molecular targets that

${ }^{*}$ Current institution: Assistant Professor, Director of Oncology Rehabilitation Medicine for SCCC, Department of Physical Medicine and Rehabilitation, University of Miami-Miller School of Medicine, Miami, FL, USA 
are associated with cancer, deliberately chosen to interact with their target and is cytostatic, blocking tumor cell proliferation instead of cytotoxic, killing the tumor cells (2).

Under the umbrella of immunotherapy cancer treatments there are several agents that utilize different pathways to activate the immune system against cancer cells, including cytokines and checkpoint inhibitors (CPIs). CPIs increase antitumor $\mathrm{T}$-cell responses and are being used successfully in the treatment of several type of cancers. The classification of CPIs is currently based on the three checkpoint targets that can be blocked: PD-1, CTLA-4, and programmed death-1 ligand 1 (PD-L1). PD-1 and CTLA4 are highly expressed on T cells, while PD-L1 is found on tumor cells and antigen-presenting cells (3). Binding of PD-1 to PD-L1 blocks T-cell mediated immune attack of cancer cells. Monoclonal antibodies that target either PD-1 or PD-L1 can block this binding and enhance the immune response against cancer cells (4).

Melanoma, non-small cell lung, kidney, bladder, head and neck cancers, and Hodgkin lymphoma are among the cancer types that CPIs have been shown to treat. Specifically, IgG4-monoclonal antibodies that target PD-1 (nivolumab and pembrolizumab) have shown promise in treating advanced cancers by dampening the PD-1/PD-L1 immuno-inhibitory events $(5,6)$. However, CPIs are also associated with immune attack toward normal cells, and can promote autoimmunity. Recent literature cites inflammatory effects related to immune-related adverse events (irAEs). PD-1 inhibitors have been linked to autoimmune disorders affecting either the central or peripheral nervous system, with an estimated frequency of neurological autoimmunity in PD-1 inhibitor treated patients $2.9-4.2 \%(5,7,8)$.

We report two cases seen by supportive care specialists with de novo myasthenia gravis and myositis associated to the use of nivolumab and their functional outcomes. These cases describe the functional impact of the rapidly progressive musculoskeletal weakness associated immunotherapy for the treatment of cancer and demonstrate the course of treatment and impact on the quality of life of these two patients.

We present the following article in accordance with the CARE reporting checklist.

\section{Case presentation}

\section{Patient 1}

\section{Clinical findings}

A 65-year-old male with a history of metastatic clear cell renal cell cancer with metastasis to the lungs was in his usual state of health until one week prior to admission. He had received his $4^{\text {th }}$ cycle of nivolumab (480 $\mathrm{mg}$ every 4 weeks) with new addition of sitravatinib (120 mg po daily) and noticed the next day, unusual neck weakness, with the inability to hold up his head, double vision, trouble breathing when laying down, and symptoms of urinary incontinence (Figure 1A). On physical examination in the emergency room, the fatigability test was positive for worsened ptosis, limited adduction of the left eye with diplopia in 5 seconds after sustained up-gaze, and proximal weakness in bilateral upper extremities. His negative inspiratory force was $-40 \mathrm{cmH}_{2} \mathrm{O}$, and his vital capacity was $2.2 \mathrm{~L}$. Manual muscle testing revealed proximal and distal muscle weakness of grades $4 / 5$ and $5 / 5$, respectively.

\section{Diagnostic assessments}

He was admitted to the intensive care unit (ICU) due to concern of the onset of myasthenia gravis (MG) or possible acute inflammatory demyelinating polyradiculoneuropathy (AIDP). Labs revealed elevated levels of creatine kinase (CK; 2,299 $\mathrm{ng} / \mathrm{L})$ and troponin $\mathrm{T}(1,351 \mathrm{ng} / \mathrm{mL})$. Nerve conduction study (NCS) with repetitive stimulation and Electromyography (EMG) performed on day 2 of hospitalization suggested post neuromuscular junction (NMJ) seen in MG (Tables 1-3). Repetition stimuli testing showed post exercise decrement with muscle fatigue (Table 2). These findings were seen despite administration of pyridostigmine. Additional lab work revealed the presence of acetylcholine receptor antibodies (AChR-Abs), myositis antibodies, and striated muscle antibodies.

\section{Therapeutic interventions}

On admission to the ICU, the patient was immediately started on methylprednisolone $(1 \mathrm{mg} / \mathrm{kg} / \mathrm{day})$ and pyridostigmine (30 $\mathrm{mg}$ three times a day) and remained on methylprednisolone, pyridostigmine, and intravenous immunoglobulin (IVIG) for 5 days. He made improvements in function and strength, his neck weakness resolved, and CK levels continued to trend down. He was assessed and continued with physical and occupational therapy sessions.

\section{Follow-up and outcomes}

After receiving the above treatment regimen, along with physical and occupational therapy sessions, his functional status improved. He had improvement in his ability to transfer from minimal assistance to supervision level and was ambulating 350 feet without an assistive device. He was 
A
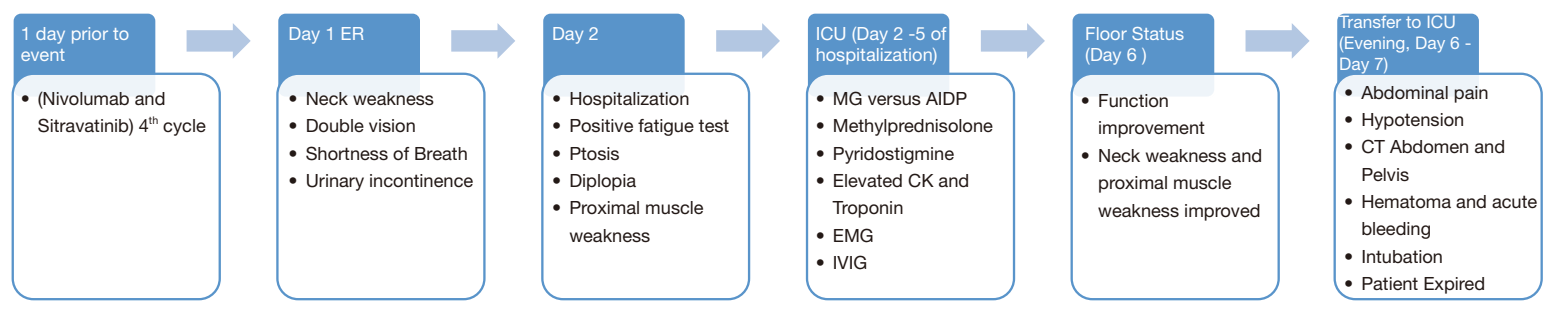

B
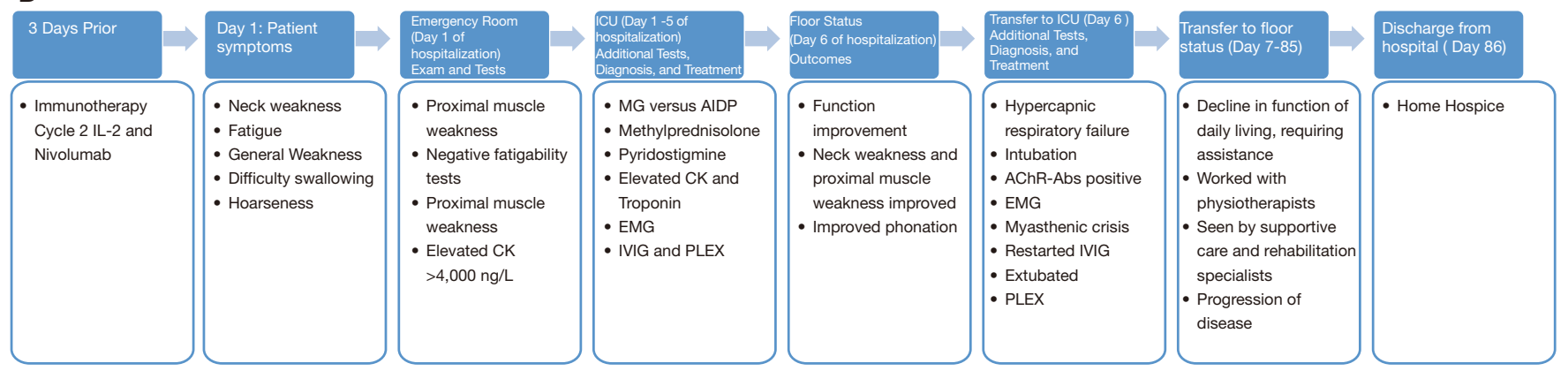

Figure 1 Timeline events of patient 1 (A) and patient 2 (B).

Table 1 Patient 1 nerve conduction study

\begin{tabular}{|c|c|c|c|c|}
\hline Nerve and site & Latency & Amplitude & Conduction velocity & F-wave latency \\
\hline \multicolumn{5}{|l|}{ Motor conduction } \\
\hline Peroneal, B & Absent & Absent & Absent & Minimally prolonged \\
\hline $\begin{array}{l}\text { Extensor absent digitorum } \\
\text { brevis (EDB), B }\end{array}$ & Absent & Absent & Absent & Minimally prolonged \\
\hline Left median, L & Mildly prolonged & Normal & Normal & Minimally prolonged \\
\hline Ulnar, L & Mildly prolonged & Normal & $\begin{array}{l}\text { Mild slowing in the } \\
\text { forearm segment }\end{array}$ & Minimally prolonged \\
\hline Superficial peroneal, B & Absent & Absent & Absent & \\
\hline Median, L & $\begin{array}{l}\text { Moderately } \\
\text { prolonged }\end{array}$ & Moderately low & Moderately slow & \\
\hline Median, $\mathrm{R}$ & $\begin{array}{l}\text { Moderately } \\
\text { prolonged }\end{array}$ & Moderately low & Moderately slow & \\
\hline Ulnar, L & Absent & Absent & Absent & \\
\hline Ulnar, R & Normal & Mildly reduced & Moderately slow & \\
\hline Radial, L & Normal & Moderately reduced & Moderately reduced & \\
\hline
\end{tabular}


Table 2 Patient 1 repetitive nerve stimulation study

\begin{tabular}{lll}
\hline Type of stimuli & 5 stimuli, at 2 hertz & 7 stimuli, at 2 hertz \\
\hline Muscle & & \\
Nasalis/facial & Could not tolerate & \\
Accessory nerve recording trapezius & No decrement & Post exercise decrement maximum 14\%, corrected at 2.5 minutes \\
Left peroneal nerve recording tibialis anterior & No decrement & No incremental change \\
\hline
\end{tabular}

Table 3 Patient 1 electromyography (EMG) study

\begin{tabular}{|c|c|c|c|c|c|c|c|c|c|}
\hline Muscle* $^{*}$ & $\begin{array}{l}\text { Insertional } \\
\text { activity }\end{array}$ & Fibrillation & $\begin{array}{l}\text { Positive } \\
\text { sharp wave }\end{array}$ & Fasciculation & Duration & Amplitude & $\begin{array}{l}\text { Polyphasic } \\
\text { wave }\end{array}$ & Configuration & Recruitment \\
\hline ADM & Normal & None & None & None & Normal & Increased & None & Normal & $\begin{array}{l}\text { Moderately } \\
\text { reduced }\end{array}$ \\
\hline Vastus femoris & Normal & None & None & None & Increased & Increased & None & Normal & $\begin{array}{l}\text { Moderately } \\
\text { reduced }\end{array}$ \\
\hline
\end{tabular}

*Deltoid, biceps, triceps, lliopsoas muscles were tested and found to be normal.

deemed safe to go home with his family. Unfortunately, prior to discharge home, on day 6 of acute hospitalization, the patient complained of abdominal pain with hypotension. He was transferred to the ICU after a computed tomography (CT) scan of the abdomen/pelvis revealed a large right pelvic hematoma measuring $15 \mathrm{~cm}$. He underwent emergent embolization of the right epigastric artery and was intubated for the procedure. He was extubated on day 7 in the ICU and failed attempts to wean off the ventilator. He desaturated and became bradycardic with a heart rate 20 beats per minute. The patient was changed to comfort care, was taken off the ventilator, and subsequently passed.

\section{Patient 2}

\section{Clinical findings}

A 83-year-old male with history of metastatic urothelial carcinoma was receiving treatment with pegylated interleukin- (IL-2) and nivolumab (480 $\mathrm{mg}$ every 4 weeks). Three days after receiving the second cycle of immunotherapy, he became weak, with difficulty holding his head up, difficulty swallowing, generalized weakness and new hoarseness (Figure 1B). He reported his symptoms were worse at the end of day. He denied any double vision.
On admission, physical exam showed a frail appearing man with dry mucous membranes and hoarse voice, extraocular muscles were intact, and a midline tongue. Sustained upgaze for 60 seconds was normal and did not show signs of fatigability. Strength exam showed neck flexion/extension graded $5 / 5$, proximal muscle weakness with deltoids graded $4+/ 5$, iliopsoas $4+/ 5$, otherwise distally $5 / 5$. There was no tenderness among his large muscle groups and no fatigability with maximal exercise of the deltoids with 10 repetitions. Reflexes were 1+ throughout, with downgoing toes. Sensation to touch and proprioception were intact distally.

\section{Diagnostic assessments}

Admission labs were abnormal including CK greater than $4,000 \mathrm{ng} / \mathrm{L}$ and aldolase elevated at 55.6 U/L. A muscle biopsy of the rectus femoris muscle showed focal perimysial chronic inflammatory cell infiltrate (Figure 2). An EMG performed on day 2 of hospitalization suggested generalized myopathy with irritable features (fibrillations and positive sharp waves), most likely a result of an inflammatory/ reactive process, but there was no definite evidence of a neuromuscular transmission disorder (such as MG). Based on laboratory reports, EMG, and muscle biopsy, he was diagnosed with myositis. 


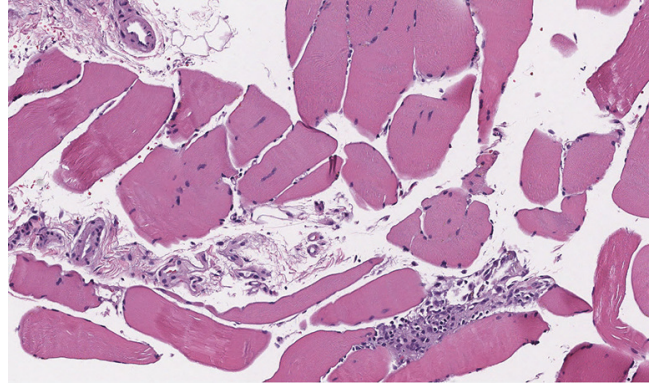

Figure 2 Skeletal muscle and fibroconnective tissue with focal perimysial chronic inflammatory cell infiltrate (left rectus femoris). Hematoxylin and Eosin (H\&E) staining, 10× magnification.

\section{Therapeutic interventions}

He received methylprednisolone and plasma exchange (PLEX) for 5 days. He showed improvement in strength, and improved phonation without having to communicate using the white board. However, that same night he developed hypercapnic respiratory failure requiring intubation.

He was diagnosed with MG based on positive AChRAbs, although repeated EMGs did not show evidence of NMJ dysfunction. He was diagnosed with myasthenic crisis. Due to hypotensive shock, PLEX was discontinued and instead received a repeat dose of IVIG with significant clinical improvement and mechanic ventilation discontinuation. In total, he received 2 rounds of PLEX and 2 rounds of 5 days IVIG, tacrolimus, and steroids throughout his hospitalization.

\section{Follow-up and outcomes}

Functionally, on admission, patient was at supervision level (FIM 5) with bed mobility, transfers and gait 150 feet with Assistive Device. He required total assist with eating and moderate assist with lower body dressing (FIM 3). During his 3-month hospitalization he had functional decline despite physical and occupational therapy sessions, with transfers and gait requiring max assist (FIM 2). He was dependent with tube feeds (FIM 1) and lower body dressing (FIM 1). Due to his functional and medical decline, and evidence of progression of the disease, the patient went home with hospice care. After 1 month under hospice care, the patient died.

\section{Discussion}

We highlight two cases of skeletal weakness (de novo MG and myositis) related to cancer immunotherapy treatment. In the first case, it should be noted that myasthenia does not commonly include elevated CK and troponin levels, but the confirmation of the MG diagnosis was made through EMG findings for post NMJ dysfunction with repetition stimuli showing post exercise decrement with muscle fatigue and positive AChR-Abs. This demonstrates that immunotherapy-induced skeletal muscle weakness can present with multiple symptoms that could suggest multiple diagnoses. It is important to carefully assess patients for both muscle involvement (such as myositis) and neuromuscular abnormality (such as MG). One of the diagnostic challenges for clinicians is that these patients might not present with a single diagnosis alone but could possibly have a combination of presentations that may not fit a traditional diagnosis.

More research into the frequency of irAEs is urgently needed since immunotherapy is increasingly used in the treatment of cancer. Zimmer $e t a l$. published a study with 496 patients being treated with anti-PD-1 therapy in which there were a total of 242 irAEs in 138 patients (8). These side effects included respiratory $(17.3 \%)$, musculoskeletal $(15.2 \%)$, cardiac $(3.6 \%)$, and hematological $(0.7 \%)$ events. In a review by Makarious et al., MG was reported in 17 cases associated with anti-PD-1 inhibitor, seven cases with nivolumab and 10 cases with pembrolizumab (9). Among the seven reported cases of nivolumab-associated MG, $57.1 \%$ were de novo, $14.3 \%$ were exacerbations, and $28.5 \%$ were exacerbations of subclinical disease. The mean onset of symptoms was 5.14 weeks post initiation, with a $42.9 \%$ mortality rate. All cases of nivolumab-associated MG occurred within a relatively short time frame (2-9 weeks) from drug initiation. In above cases reported here, weakness occurred after the second and third cycles.

Although, cases of irAEs have been reported in the literature, it has not been reported in great detail the patient outcomes, prolonged morbidity, functional decline and quality of life with prolonged hospitalization. Patient 1's death was not directly due to MG crisis, but his inability to wean from the ventilator could be attributed to the overall weakness of his respiratory muscles. His overall morbidity status was high and with acute blood loss event, he was unable to recover. In patient 2, skeletal muscle weakness from myositis related to immunotherapy resulted in a prolonged hospitalization of greater than 2 months and rehabilitation course, with the patient unable to return to his prior level of independence. These examples show the 
vulnerability of the immunotherapy patient population and how irAEs could lead to prolonged hospitalizations and significant functional impairments and dependence on caregivers.

This case series provides a different perspective to the approach of immunotherapy induced musculoskeletal weakness. It focused on the functional repercussion of these side effects and how early interventions, including evaluation by a multidisciplinary team, including physiatrist, supportive care physician and physical and occupational therapy participation can improve functional outcomes. Limitations include the small number of patients included as it makes it difficult to extrapolate the results of the interventions. Additionally, these patients were under the care of a comprehensive cancer center with strong rehabilitation and supportive care services, with resources that may not be widely available in the United States.

\section{Conclusions}

With new treatment modalities emerging in the field of immunotherapy for targeting malignancies, clinicians must be aware of the importance of timing with treatment and development of irAEs. Recognition of symptoms and prompt treatment can potentially prevent prolonged impairment, reduced quality of life and associated mortality.

\section{Acknowledgments}

The authors thank the patients for their permission to publish their cases, inpatient staff that have assisted with these patients and their care and Brittany Cullen for her expert administrative assistance.

Funding: None.

\section{Footnote}

Conflicts of Interest: All authors have completed the ICMJE uniform disclosure form (available at http://dx.doi. org/10.21037/apm-20-454). EB reports grants from Helsinn Healthcare, outside of the submitted work. The other authors have no conflicts of interest to declare.

Ethical Statement: The authors are accountable for all aspects of the work in ensuring that questions related to the accuracy or integrity of any part of the work are appropriately investigated and resolved. All procedures performed in studies involving human participants were in accordance with the ethical standards of the institutional and/or national research committee(s) and with the Helsinki Declaration (as revised in 2013). The authors alone are responsible for the content and writing of the paper. Patient 1 passed away in the hospital prior to the authorship of these case reports therefore consent is not required. Patient 2 provided written informed consent for publication of this study and any accompanying images. A copy of the written consent is available for review by the Editor-in-Chief of this journal.

Open Access Statement: This is an Open Access article distributed in accordance with the Creative Commons Attribution-NonCommercial-NoDerivs 4.0 International License (CC BY-NC-ND 4.0), which permits the noncommercial replication and distribution of the article with the strict proviso that no changes or edits are made and the original work is properly cited (including links to both the formal publication through the relevant DOI and the license). See: https://creativecommons.org/licenses/by-nc$\mathrm{nd} / 4.0 /$.

\section{References}

1. Rotte A, D'Orazi G, Bhandaru M. Nobel committee honors tumor immunologists. J Exp Clin Cancer Res 2018;37:262.

2. National Cancer Institute. Immunotheray to Treat Cancer. [updated November 28, 2018]; Accessed March 25, 2020.

Available online: https://www.cancer.gov/about-cancer/ treatment/types/immunotherapy.

3. Tocheva AS, Mor A. Checkpoint Inhibitors: Applications for Autoimmunity. Curr Allergy Asthma Rep 2017;17:72.

4. Kao JC, Brickshawana A, Liewluck T. Neuromuscular Complications of Programmed Cell Death-1 (PD-1) Inhibitors. Curr Neurol Neurosci Rep 2018;18:63.

5. Liewluck T, Kao JC, Mauermann ML. PD-1 Inhibitorassociated Myopathies: Emerging Immune-mediated Myopathies. J Immunother 2018;41:208-11.

6. Sweis RF, Luke JJ. Mechanistic and pharmacologic insights on immune checkpoint inhibitors. Pharmacol Res 2017;120:1-9.

7. Kao JC, Liao B, Markovic SN, et al. Neurological Complications Associated With Anti-Programmed Death 1 (PD-1) Antibodies. JAMA Neurol 
2017;74:1216-22.

8. Zimmer L, Goldinger SM, Hofmann L, et al. Neurological, respiratory, musculoskeletal, cardiac and ocular side-effects of anti-PD-1 therapy. Eur J Cancer
2016;60:210-25.

9. Makarious D, Horwood K, Coward JIG. Myasthenia gravis: An emerging toxicity of immune checkpoint inhibitors. Eur J Cancer 2017;82:128-36.
Cite this article as: $\mathrm{Ng} \mathrm{AH}$, Molinares DM, Ngo-Huang AT, Bruera E. Immunotherapy-related skeletal muscle weakness in cancer patients: a case series. Ann Palliat Med 2021;10(2):2359-2365. doi: 10.21037/apm-20-454 